\title{
Density-dependent immunity and parasitism risk in experimental populations of lizards naturally infested by ixodid ticks
}

\author{
Marianne Mugabo, ${ }^{1,2,5}$ Samuel Perret,${ }^{3}$ Beatriz Decencière, ${ }^{3}$ Sandrine Meylan,,${ }^{1,4}$ \\ And Jean-François Le Galliard ${ }^{1,3}$ \\ ${ }^{1}$ CNRS-UPMC, UMR 7618, iEES Paris, Université Pierre et Marie Curie, 7 Quai St. Bernard, 75005 Paris, France \\ ${ }^{2}$ School of Biology, Faculty of Biological Sciences, Manton Building, University of Leeds, Clarendon Way, \\ Leeds LS29JT United Kingdom \\ ${ }^{3}$ CNRS-ENS, UMS 3194, CEREEP-Ecotron IleDeFrance, École Normale Supérieure, 78 rue du Château, \\ 77140 St-Pierre-lès-Nemours, France \\ ${ }^{4}$ ESPE de Paris-Université Sorbonne Paris IV, 10 rue Molitor, 75016 Paris, France
}

\begin{abstract}
When effective immune defenses against parasites are costly and resources limited, individuals are expected to alter their investment in immunity in response to the risk of infection. As an ecological factor that can affect both food abundance and parasite exposure, host density can play an important role in host immunity and host-parasite interactions. High levels of intraspecific competition for food and social stress at high host density may diminish immune defenses and increase host susceptibility to parasites. At the same time, for contagious and environmentally transmitted parasites, parasite exposure often increases with host density, whereas in mobile parasites that actively search for hosts, parasite exposure can decrease with host density due to the "encounter-dilution effect." To unravel these multiple and potentially opposing effects of host density on immunity, we manipulated density of the common lizard Zootoca vivipara and measured local inflammation in response to PHA injection and levels of infestation by the tick Ixodes ricinus, a mobile ectoparasite for which we expected an encounter-dilution effect to occur. Local inflammation strongly decreased with lizard density in adults, but not in yearlings. Tick infestation (abundance and prevalence) was negatively correlated with lizard density in both age classes. Using path analyses, we found independent, direct negative density feedbacks on immunity and parasite exposure in adults, supporting the hypothesis of energy constraints and/or physiological stress acting on immunity at high density. In contrast, for yearlings, the best path model showed that density diluted exposure to parasites, which themselves down-regulated immune defenses in lizards. These results highlight the importance of investigating the pathways among host density, host immunity, and parasite infestation, while accounting for relevant individual traits such as age.
\end{abstract}

Key words: common lizard; density dependence; encounter-dilution effect; host-parasite interactions; immune defenses; inflammation; Ixodes ricinus; path analysis; Saint-Pierre-lès-Nemours, France; Zootoca vivipara.

\section{INTRODUCTION}

The immune system is one of the main defenses of individuals against parasites and diseases, along with antiparasite behaviors (Altizer et al. 2003), because it allows survival in infectious environments (Lochmiller and Deerenberg 2000, Møller and Saino 2004). However, maintaining effective immune defenses and mounting an immune response are costly and compete with other energy-demanding functions such as growth and reproduction (reviewed in Sheldon and Verhulst 1996, Lochmiller and Deerenberg 2000). As a consequence, when resources are limited, individuals should adjust their investment in parasite resistance to the risk of being infected (e.g., Møller et al. 2001, Tschirren and

Manuscript received 18 March 2014; revised 8 July 2014; accepted 22 July 2014. Corresponding Editor: K. D. Lafferty.

5 E-mail: marianne.mugabo@gmail.com
Richner 2006). Given the potential effects of host density on resource abundance and parasite transmission, the relationship between host density, hostparasite interactions, and host immunity has therefore received increasing interest from eco-immunologists over the last decade (reviewed in Rifkin et al. 2012).

The risk of parasite infections can either increase or decrease with host group size or population density (Mooring and Hart 1992, Coté and Poulin 1995, Rifkin et al. 2012). For contagious and environmentally transmitted parasites, transmission rates increase with host density (Mooring and Hart 1992, Coté and Poulin 1995, Rifkin et al. 2012) and hosts should therefore increase their investment in immunity when they live in larger groups (see examples in Rifkin et al. 2012). In contrast, in host-parasite systems where parasites actively search for hosts (e.g., biting flies or parasitoids) or where parasites wait for hosts in the external environment (e.g., ticks), hosts living in larger groups 
may benefit from an "encounter-dilution effect" as defined by Mooring et Hart (1992) at the host intraspecific level. In this situation, the risk of being parasitized diminishes with host group size or population density (reviewed in Mooring and Hart 1992, Coté and Poulin 1995, and Rifkin et al. 2012; see Keesing et al. [2006] for a different use of the term "dilution effect" in disease ecology in relation to host biodiversity at the host community level). Thus, hosts should decrease their investment in immune resistance against parasites when host density (or host diversity) increases (Sheldon and Verhulst 1996, Tschirren and Richner 2006). Alternatively, if host immunity is down-regulated by ectoparasites to the advantage of the latter, then a positive association between host density and immunity may arise due to the reduction of host parasite load with density (reviewed in Wikel 2013).

At the same time, intraspecific competition for food often decreases feeding and increases levels of social stress (e.g., Trenzado et al. 2006, Amundsen et al. 2007, Edeline et al. 2010), and thus should compromise immunity (e.g., Svensson et al. 2001, Tella et al. 2001, Hawley et al. 2006) and increase host susceptibility to parasites (e.g., Raffel et al. 2010). Given the possibility of both direct, negative effects of density on immunity through intraspecific competition and indirect effects mediated by parasite exposure, it is not surprising that many studies have found a weak or no significant association between immunity and host density, group size, or levels of intraspecific competition (see metaanalysis by Rifkin et al. 2012). Targeted study of immune performances and parasitism risks in natural situations where group size or parasite exposure are manipulated should help to reveal the relationship between host density and host-parasite interactions (e.g., Raffel et al. 2010). Furthermore, immunity can also be affected by behavioral and physiological differences among individuals due to sex, age, or social status independently from group size (e.g., Tschirren et al. 2003, Hawley et al. 2006, Raffel et al. 2010). Such state-dependent variation can give rise to substantial changes in exposure to and/or attractiveness for parasites (e.g., Christe et al. 2007, Grear et al. 2009) and ultimately in the level of parasite infestation (Altizer et al. 2003, Raffel et al. 2010). Thus, it is also necessary to account for relevant traits of hosts in order to unravel the density dependence of host-parasite interactions (Fauchald et al. 2007, Raffel et al. 2010).

In this study, we manipulated density in experimental populations of the common lizard Zootoca vivipara maintained in seminatural conditions and measured density-dependent effects on immune defenses and levels of parasite infestation by the tick Ixodes ricinus, while preventing interactions with other host species of $I$. ricinus. In this mobile ectoparasite of mammals, birds, reptiles, and amphibians, individuals actively search for or wait for their host in the vegetation. Each life stage (larva, nymph, and adult female) feeds on one host for a few days before dropping to the ground to transition to the next stage or to lay eggs (Gray 1991). I. ricinus is thus a good candidate for the occurrence of an encounter-dilution effect (e.g., Krasnov et al. 2007). Larvae and nymphs of I. ricinus are the predominant mobile ectoparasites infecting common lizards in our study site (Saint-Pierre-lès-Nemours, France) and represent a potential fitness cost for lizards (e.g., Heylen and Matthysen 2008). We assessed immune defenses by local inflammation, which is the first line of defenses against blood-feeding ectoparasites (e.g., Tschirren et al. 2007, Owen et al. 2009).

We first characterized age- and sex-specific patterns of density dependence in local inflammation and tick infestation. Using path analyses (Shipley 2009), we then investigated the relationships between lizard density, tick infestation, and local inflammation. We recently demonstrated negative density feedbacks for body growth and reproduction, with stronger effects in females than in males and stronger effects in juveniles than in adults (Mugabo et al. 2013). In addition, we previously reported that food deprivation has different long-term effects on local inflammation in growing vs. fully grown individuals (Mugabo et al. 2010, 2011). Thus, we expected (1) an encounter-dilution effect of $I$. ricinus parasitism risk with lizard density as predicted for mobile ectoparasites (Mooring and Hart 1992); (2) a direct negative effect of lizard density on local inflammation due to increased intraspecific competition, with age- and sex-specific responses, as in our previous demographic studies; and/or (3) an indirect effect of lizard density on local inflammation mediated by the density-dependent parasitism risk. If lizards adjust their investment in parasite resistance to the risk of being infected (i.e., adaptive plasticity; Sheldon and Verhulst 1996), then local inflammation should decrease with lizard density, along with the risk of infection. Yet, if ticks down-regulate host immune responses (Wikel 2013), a positive association could arise between lizard density and local inflammation due to the reduction of tick infestation with the dilution effect.

\section{Material and Methods}

\section{Model species and manipulation of population density}

The lizard Zootoca vivipara is a small (adult snoutvent length, SVL, is $50-75 \mathrm{~mm}$ ), ovoviviparous species inhabiting humid habitats across northern Eurasia (see Plate 1). Natural populations can be divided into three age classes: juveniles (newborns of the year), yearlings (1-2 years old), and adults ( $\geq 2$ years old; Massot et al. 1992). During the summer 2008, we manipulated the initial density of 24 experimental populations maintained in $10 \times 10 \mathrm{~m}$ outdoor enclosures located in a natural meadow $\left(48^{\circ} 17^{\prime} \mathrm{N}, 2^{\circ} 41^{\prime} \mathrm{E}\right.$, Saint-Pierre-lèsNemours, France). Enclosures provided lizards with natural food, shelters, and basking sites and were closed to ungulates (important hosts for I. ricinus nymphs and adult females; Mejlon 2000) by fences, but could 
occasionally be accessed below ground by intrusive small mammals (important hosts of I. ricinus larvae; Mejlon 2000). These intrusions were rare and opportunistic and occurred independently of lizard density; therefore, they should have had a negligible impact on levels of tick infestation of lizards.

Populations were established post-breeding following a gradient of five density levels ranging from 7 to 35 adults and yearlings (equivalent to 700 to 3500 individuals/ha) together with 10 to 50 juveniles. Density level 1 had 1 adult male, 2 adult females, 2 yearling males, 2 yearling females, and 5-6 and 4-5 juvenile males and females, respectively. Density levels 2 to 5 differed from density level 1 by a multiplicative factor of 2 to 5, respectively, and had similar age and sex structures. The number of population replicates was adjusted to reach a similar sample size per density level (level $1=10$ replicates, level $2=5$ replicates, level $3=4$ replicates, level $4=3$ replicates, and level $5=2$ replicates). Enclosures were randomly assigned to density levels (for more details about the experimental design, see Mugabo et al. 2013). The intermediate level of density (level 3, 21 adults and yearlings) matched the pre-breeding density at equilibrium in our study site (Lecomte et al. 2004, Mugabo et al. 2013). Natural populations from where individuals originate (Mont Lozère, southern France, $44^{\circ} 27^{\prime}$ N, $3^{\circ} 44^{\prime}$ E) have mean densities ranging from 200 to 2000 adults and yearlings/ ha and are characterized by less favorable environmental conditions (temperature, food availability, and predation risk) than in our study site (Massot et al. 1992, Lecomte et al. 2004, González-Suárez et al. 2011).

Lizards $(n=378$ adults and yearlings and 549 juveniles) were randomly assigned to experimental populations and were released in outdoor enclosures in June-July 2008 after removing all ticks. The preexperimental local inflammation was assessed prior to release in adults and yearlings and did not differ between treatments and sexes $(F$ tests, all $P>0.16)$. Preexperimental local inflammation could not be assessed in juveniles because of their small body size $(\mathrm{SVL}<30$ $\mathrm{mm}$ ). All surviving lizards were recaptured in late spring 2009. In our analysis, we classified lizards according to two age classes at recapture in 2009: adults (age $>1$ year in 2009, $n=101$ ) and yearlings (aged 1 year in 2009, $n=$ 202). After recapture, we measured the postexperimental local inflammation, and tick infestation was estimated by tick prevalence (proportion of parasitized individuals) and abundance (number of ticks per individual). Parasite prevalence and abundance represent two complementary aspects of infection and are often correlated (e.g., Simkova et al. 2002, Krasnov et al. 2007). However, they may be affected by different intrinsic (e.g., age, sex, behavior, genetic susceptibilities) and extrinsic (e.g., parasite exposure, habitat quality) factors (Krasnov et al. 2007), and can therefore vary in their response to host density (e.g., Viljoen et al. 2011).

\section{Estimation of local inflammation}

Local inflammation was assessed by skin swelling in response to the injection of a mitogen, the phytohemagglutinin (PHA; Sigma-Aldrich, St. Louis, Missouri, USA), as described by Mugabo et al. (2010). Skin swelling was calculated as the absolute difference in the thickness of the leg (to the nearest $0.01 \mathrm{~mm}$ ) $12 \mathrm{~h}$ after (evening, between 21:00 and 22:30 hours) and just before (morning, between 08:40 and 10:40 hours) the PHA injection (e.g., Meylan et al. 2013). Time at injection, duration between the two measurements, and body size had no effect on local inflammation (postexperimental measurements; likelihood ratio tests, LRT: all $P>$ $0.34)$. The PHA-induced skin swelling is used in ecoimmunology to evaluate the ability of an individual to mount a local inflammation in non-model species (Vinkler et al. 2010, Demas et al. 2011, Pedersen and Babayan 2011) and can be negatively correlated with ectoparasite fitness (e.g., Christe et al. 2000, Tschirren et al. 2007, Owen et al. 2009). PHA injection triggers a local hemagglutination and leukocyte infiltration and involves both adaptive (mediated by T-lymphocytes) and innate (mediated by other leukocytes such as granulocytes and macrophages) components of the immune system (Martin et al. 2006, Turmelle et al. 2010, Vinkler et al. 2010, Brown et al. 2011). The blood cell proliferation and the associated skin swelling in response to PHA are dynamic processes, with peak cell responses varying among leukocyte populations, between $12 \mathrm{~h}$ and $48 \mathrm{~h}$ postinjection (Martin et al. 2006, Turmelle et al. 2010, Brown et al. 2011).

To validate this protocol independently from our density manipulation, we tested the kinetics of the local inflammation after a first PHA injection and the dependence of local inflammation on a previous exposure to PHA (acquired immunity; see Appendix A). In a first pilot study, we injected 20 lizards with a control PBS solution (phosphate buffered saline; SigmaAldrich, St. Louis, Missouri, USA) and 38 lizards with PHA, and measured difference in the thickness of the leg over time from 12 to $48 \mathrm{~h}$ every $12 \mathrm{~h}$. This demonstrated a maximum swelling response at $12 \mathrm{~h}$, a regular decrease of swelling with time, and a significant interindividual variation among lizards injected with PHA, but not with PBS (see Appendix A: Fig. A1.A). In a second pilot study, we compared the second swelling response of 24 lizards first injected with PHA 7 days earlier and of 21 lizards first injected with PBS. We measured differences in the thickness of the leg from 12 to $36 \mathrm{~h}$ every $12 \mathrm{~h}$ after the PHA injection. This assay was proposed by Brown et al. (2011) to test for the activation of acquired cell-mediated immunity against PHA after a secondary exposure, in addition to the components of innate and adaptive primary cell-mediated immunity activated at each exposure (Martin et al. 2006, Turmelle et al. 2010, Vinkler et al. 2010, Brown et al. 2011). As expected, this test demonstrated a larger secondary response to PHA injection than the primary response (Appendix A: Fig. 
A1.B), as well as uncorrelated primary and secondary responses at the peak of the inflammation $(12 \mathrm{~h}$ post injection; Pearson's product-moment correlation coefficient $=0.25 ; 95 \% \mathrm{CI}=[-0.17,0.59] ; t=1.20, \mathrm{df}=22, P=$ $0.24)$. Altogether, these pilot studies justify a measurement of peak local inflammation around $12 \mathrm{~h}$ after injection, and are consistent with a joint activation of innate immunity and cell-mediated immunity in the common lizard in response to PHA.

\section{Statistical analyses}

Density dependence in postexperimental local inflammation was examined with mixed-effects linear models in $\mathrm{R}$ version 3.0.2 ( $\mathrm{R}$ Development Core Team 2013) following Bates (2010). Density dependence in tick prevalence (presence/absence, binomial distribution) and abundance (number of ticks, Poisson distribution) was examined with mixed-effects generalized linear models following Bolker et al. (2009). Enclosure identity was included in all models as a random effect. The fixed part of all models included a linear density effect, a sex effect, an age class effect (adults vs. yearlings), and their first-order interactions, as well as an effect of time since release. In addition, we investigated the relationship between the preexperimental (prior release in 2008) and the postexperimental (after recapture in 2009) local inflammation in all adults tested in both years $(n=101)$, with local inflammation included in a linear mixed-effect model as the response variable, and the PHA injection session (pre- vs. post-) in interaction with sex as explanatory variables. Individual identity was included in the model as a random effect nested in enclosure identity. The Pearson's product moment correlation coefficient between the two measurements was also estimated in males and in females.

We used a maximum likelihood approach for normally distributed responses and a Laplace approximation of the maximum likelihood for non-normally distributed responses in the lmer procedure (Bolker et al. 2009, Bates et al. 2013). We tested for the significance of fixed effects with LRT tests (Bolker et al. 2009) instead of using marginal $F$ tests because the use and calculation of degrees of freedom in marginal $F$ tests raises concerns (see, Faraway 2013). Associated $P$ values of LRT were obtained by implementing parametric bootstraps following Faraway (2006, 2013), with 1000 bootstrap replicates. A minimum adequate model was obtained by backward elimination of nonsignificant terms. Assumptions of normality were fulfilled, but a Bartlett test revealed significant variance heterogeneity between sexes when analyzing postexperimental local inflammation (Bartlett's $K^{2}=10.51$, df $=1, P=0.001$ ). Currently, the lmer procedure does not model heterogeneity of variance (Bates et al. 2013). However, estimates of fixed and random effects produced by the lmer procedure were very similar to those produced by the lme procedure correcting for the heterogeneity of variance. Tick abundance showed evidence of overdispersion (ratio between the sum of squared Pearson residuals and residual degrees of freedom, $\hat{c}=3.29$ ). This was accounted for by including an observation-level random effect in the models, nested in the enclosure identity random effect (i.e., Poisson-lognormal models; see Elston et al. 2001). Similar qualitative results were obtained for tick abundance using a negative binomial distribution. However, mixed-effects generalized linear models with a negative binomial distribution are currently an experimental feature of the glmer procedure (Bates et al. 2013). Parameter estimates of generalized linear models are provided on the scale of the canonical link function (i.e., log scale in Poisson regressions and logit scale in logistic regressions).

Finally, we studied the relationship between lizard density, local inflammation, and tick abundance using the $d$-separation test, a generalization of path analyses (Shipley 2009). Prevalence was not included in path analyses because prevalence and abundance were correlated (Appendix B). We compared three scenarios: in path model 1 , density had indirect effects on local inflammation through direct effects on tick abundance; in path model 2, density had indirect effects on tick abundance through direct effects on local inflammation, i.e., tick abundance was a response of density-dependent host immunity; and in path model 3 , density independently affected local inflammation and tick abundance (Appendix B: Fig. B1). Path models were rejected or not based on the $C$ statistic associated with the $d$-separation test, and support received by alternative path models was estimated using Akaike's information criterion (Burnham et al. 2011). This method of path analyses accounts for the hierarchical structure of the data and the non-normal distribution of tick abundance (see Appendix B for details).

\section{RESUlts}

All parameter estimates are provided \pm SE unless otherwise stated. Local inflammation decreased linearly with density in adults, but not in yearlings (Fig. 1; for density $\times$ age class, LRT (likelihood ratio test $)=6.21, P$ $=0.012$; slope estimate is $-0.013 \pm 0.005$ in adults and $0.003 \pm 0.007$ in yearlings). As a consequence, adults had a higher local inflammation than yearlings at low, but not at high, densities. Density dependence was similar in males and in females (for density $\times$ sex, LRT $=$ $0.04, P=0.85$ ), but local inflammation was lower (LRT $=10.45, P=0.001)$ in males. When we compared the postexperimental and preexperimental local inflammation (only measured in adults), we found higher values after the experiment in adult females (preexperimental $=$ $0.07 \pm 0.009 \mathrm{~mm}$, postexperimental $=0.11 \pm 0.01 \mathrm{~mm})$, but lower values in adult males (preexperimental $=0.10$ $\pm 0.02 \mathrm{~mm}$, postexperimental $=0.02 \pm 0.02 \mathrm{~mm}$; for injection session $\times$ sex, LRT $=13.87, P<0.0001)$. Pre- and postexperimental local inflammation were not correlated (Pearson's product-moment correlation coefficient for females is $r=0.15,95 \% \mathrm{CI}=[-0.07,0.36] ; t=$ 


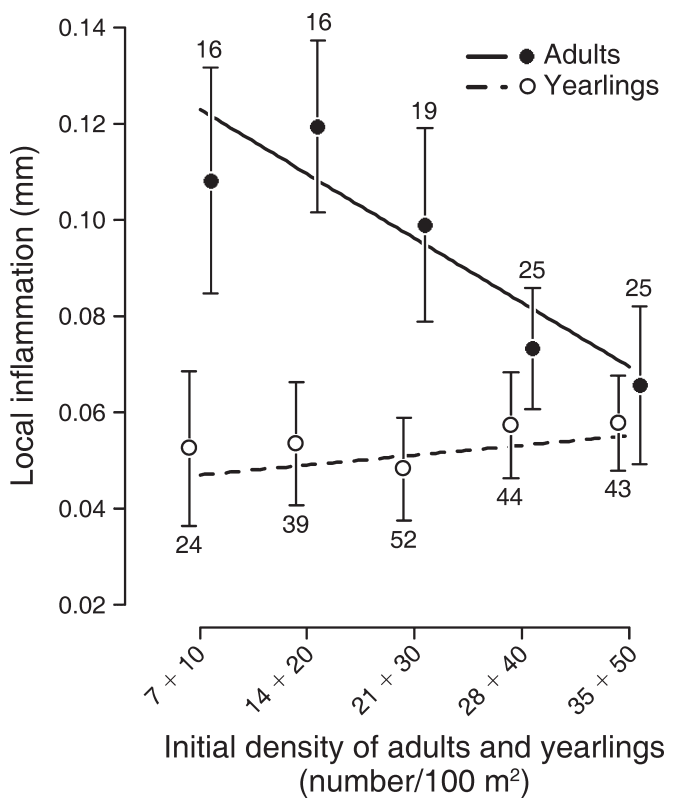

FIG. 1. Density dependence (mean \pm SE) of local inflammation in yearling and adult common lizards (Zootoca vivipara), according to initial density of adults and yearlings (age class at recapture in 2009). Numbers around error bars indicate sample sizes. Postexperimental local inflammation (i.e., a year after the manipulation of density), was assessed by the skin-swelling $12 \mathrm{~h}$ after an injection of the mitogen phytohaemagglutinin (PHA), as described by Mugabo et al. (2010). On the $x$-axis, the initial density is represented as the number of adults + yearlings (in this order) released in each $100-\mathrm{m}^{2}$ enclosure. Estimates of regression lines were obtained from the minimum adequate model in the local inflammation analysis without the additive effect of sex
1.36, $\mathrm{df}=76, P=0.18$; for males, $r=0.17[-0.24,0.56] ; t$ $=0.87$, df $=21, P=0.39$ ).

Tick prevalence (proportion of lizards parasitized) decreased strongly with density in females but not in males (Fig. 2; for density $\times$ sex, LRT $=4.61, P=0.04$; the slope estimate on the logit scale in males is $-0.49 \pm$ 0.38 and in females is $-1.27 \pm 0.34$ ). Adults and yearlings were similarly affected by density (for density $\times$ age class, $\mathrm{LRT}=0.71, P=0.45)$, but prevalence was higher in adults (Fig. 2; LRT $=8.77, P=0.005$ ). Tick abundance also decreased significantly with density (Fig. 3; $\mathrm{LRT}=5.03, P=0.03$; slope estimate on the log scale is $-0.20 \pm 0.08$ ), and was similarly affected by density among sexes and age classes (for density $\times$ sex, LRT $=$ $0.74, P=0.40$; for density $\times$ age class, $\mathrm{LRT}=0.67, P=$ $0.46)$. Tick abundance was also higher in males than in females (male contrast on the $\log$ scale is $0.28 \pm 0.12$; $\mathrm{LRT}=5.53, P=0.02)$ and in adults than in yearlings (yearling contrast on the log scale is $-0.56 \pm 0.12$; LRT $=22.15, P<0.0001)$.

We ran separate path analyses in adults and yearlings to investigate the relationship between density, local inflammation, and tick infestation because age classes strongly differed in the density dependence of local inflammation (for analyses, see Appendix B: Table B1). In adults, the path models 2 and 3 were not rejected based on the $C$ statistic (Table B1). However, model 2 was slightly less supported than model 3 and could be rejected due to the nonsupported direct path between local inflammation and abundance (Fig. 4A). Density negatively affected local inflammation through a direct path, independently of a direct negative effect of density

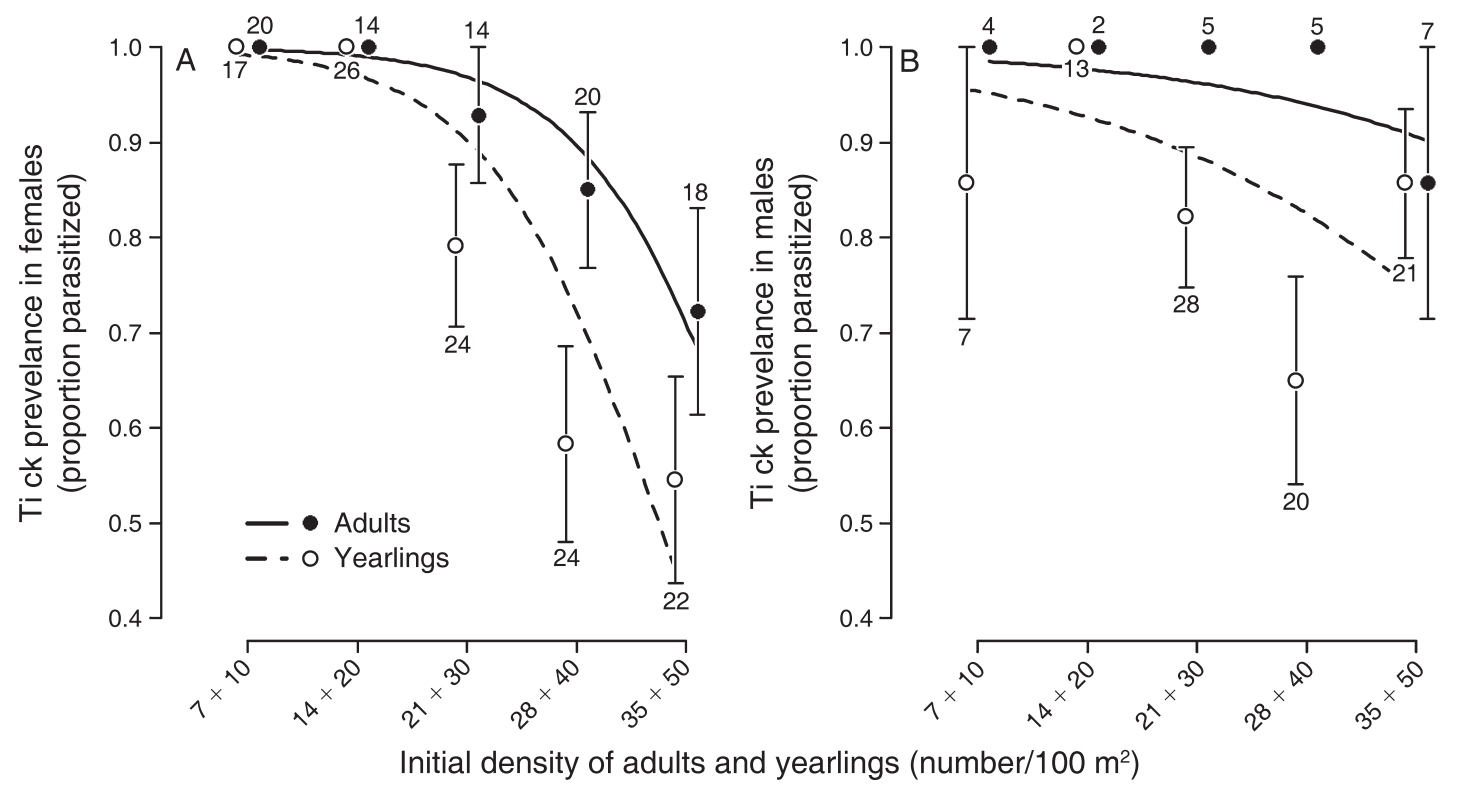

FIG. 2. Prevalence of ticks, Ixodes ricinus (mean $\pm \mathrm{SE}$ ), according to initial density of lizards (adults plus yearlings) and age class in (A) female and (B) male lizards. Numbers around error bars indicate sample size in each group. Estimates of regression lines were obtained from the minimum adequate model in the prevalence analysis. 


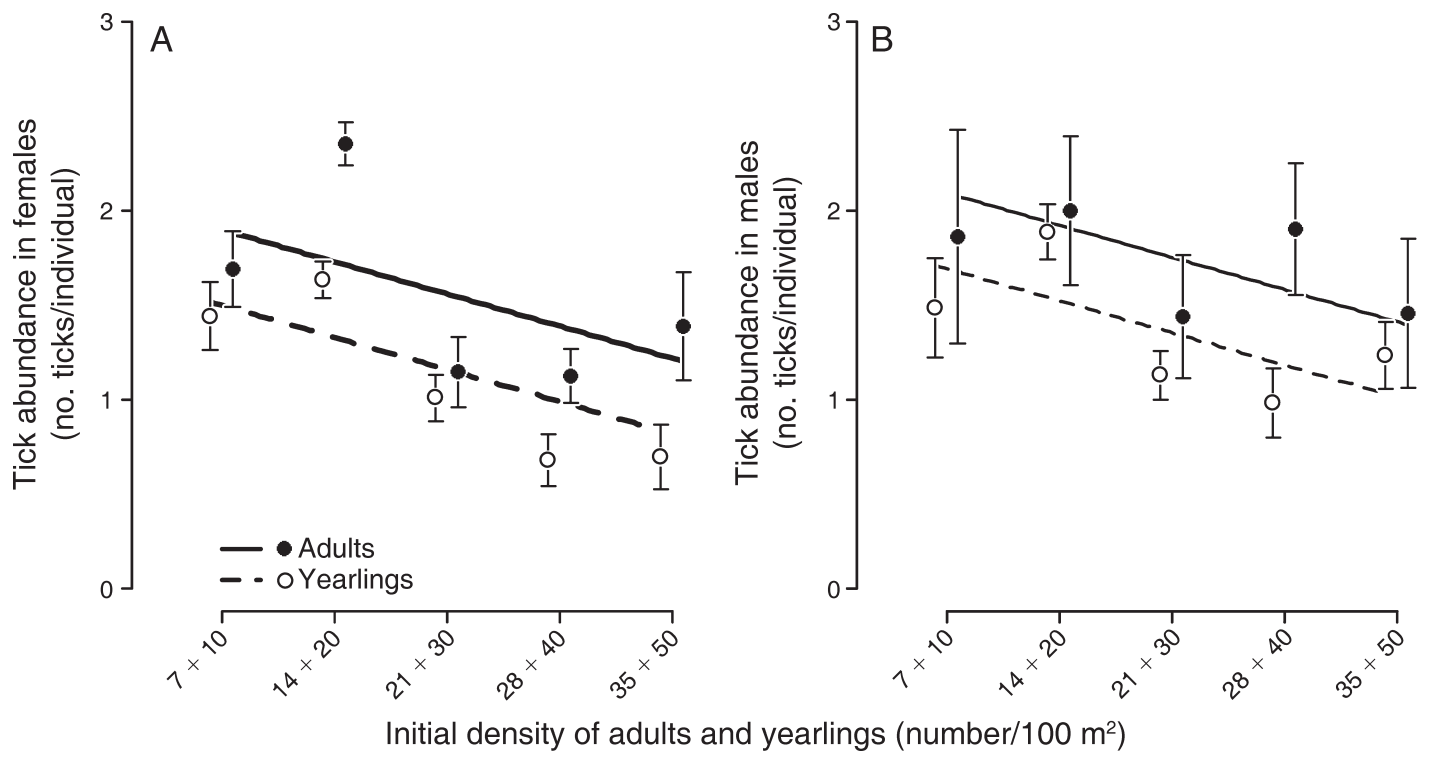

FIG. 3. Tick abundance per individual host (mean \pm SE) according to initial density of adults plus yearlings and age class in (A) female and (B) male lizards. Sample size in each group is as in Fig. 2. Estimates of regression lines were obtained from the minimum adequate model in the abundance analysis with $\log ($ tick abundance +1$)$ as the response variable.

on tick abundance (Fig. 4A and Appendix B). In yearlings, the path model 1 was the only non-rejected model based on $C$ and the only supported model based on $\mathrm{AIC}_{\mathrm{c}}$ (Appendix B: Table B1). Lizard density had direct negative effects on tick abundance, and tick abundance directly and negatively affected local inflammation (Fig. 4B and Appendix C). The indirect effect of population density on local inflammation (calculated as in Shipley 2000) was negligible (standardized estimate $=$ 0.07).

A) Adults

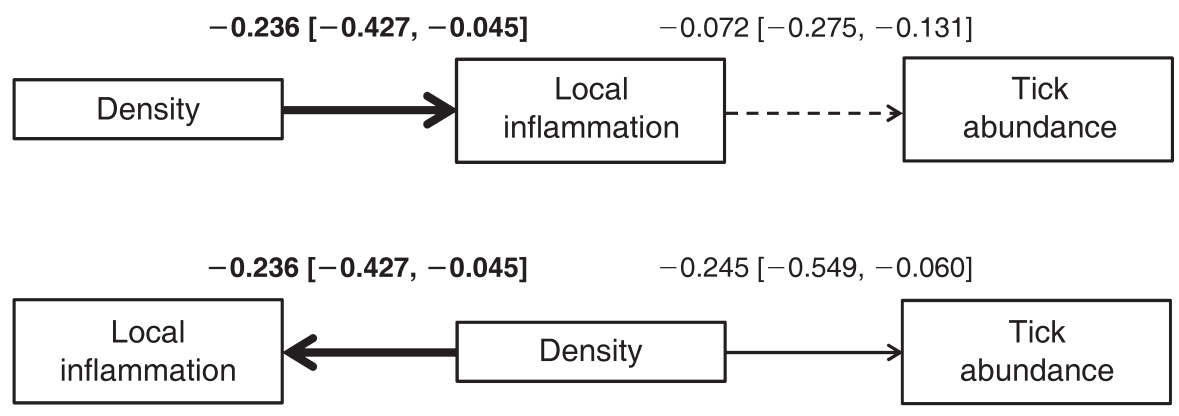

B) Yearlings

$-0.298[-0.494,-0.103]$ $-0.247[-0.383,-0.111]$

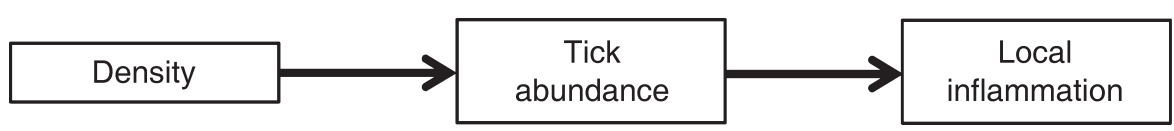

FIG. 4. Path graphs of the relationship between density, local inflammation, and tick abundance in (A) adult and (B) yearling lizards. Thick arrows indicate supported path coefficients (95\% CIs do not include 0$)$, the thin arrow indicates marginally supported path coefficients $(95 \%$ CI marginally includes 0$)$, and the dashed arrow indicates a nonsupported path. Standardized regression coefficients were obtained using linear and generalized mixed-effects models (Shipley 2009). Regression coefficients and confidence intervals for tick abundance are provided on the $\log$ scale (i.e., on the scale of the log canonical link function in Poisson regressions). We compared three scenarios: in model 1, density had indirect effects on local inflammation through direct effects on tick abundance; in model 2, density had indirect effects on tick abundance through direct effects on local inflammation, i.e., tick abundance was a response of density-dependent host immunity; and in model 3, density independently affected local inflammation and tick abundance. (A) In adults, model 2 (top) and model 3 (bottom) were both non-rejected and equally supported based on the $C$ statistic and $\mathrm{AIC}_{\mathrm{c}}$ (Appendix B: Table B1). However, model 2 was rejected because of the nonsupported direct path between local inflammation and abundance. (B) In yearlings, model 1 was the only non-rejected and supported model. 


\section{DisCUSSION}

We investigated the density dependence of local inflammation and levels of infestation by the tick Ixodes ricinus in experimental populations of common lizards Zootoca vivipara maintained in seminatural conditions. Our study yields three major results about the relationships between host density, host immunity, and host-parasite interactions. First, the PHA-induced skin swelling was negatively affected by lizard density in adult, but not in yearling, lizards. Second, the observed decrease of tick infestation with lizard density provided strong evidence of an encounter-dilution effect of the risk of parasitism by I. ricinus. Third, our path analyses supported a distinct relationship between density, local inflammation, and tick infestation that differed in yearlings vs. adults. If not accounted for, these substantial differences between age classes could prevent the detection of an association between host density, immunity, and levels of parasite infection.

\section{Age-specific local inflammation and the effect of intraspecific competition}

Local inflammation measured at the end of the experiment strongly decreased with density in adults but not in yearlings. In our study, skin swelling measurements in yearlings were obtained after a primary injection of PHA, whereas adults had already been injected with PHA prior to the experiment. Thus, agespecific responses of local inflammation could reflect the activation of different components of the immune system between the first and the second encounter with PHA. Indeed, skin swelling involves innate immunity and adaptive primary cell-mediated immunity at each exposure to PHA, but can only involve acquired cell-mediated immunity against PHA from a secondary exposure onward (Martin et al. 2006, Turmelle et al. 2010, Brown et al. 2011). This hypothesis was supported by the stronger skin swelling after the second exposure to PHA in the laboratory experiment, as well as by the lack of correlation between the primary and the secondary responses to PHA in our laboratory tests and in adults from the field experiment (as in Brown et al. 2011). Detailed histological studies of cell proliferation in the injected area would have enabled us to quantify the contribution of acquired components to the secondary inflammation (Turmelle et al. 2010, Brown et al. 2011). Unfortunately, such histological studies would require tissue biopsies at the site of injection, and were not feasible during our field experiment.

Assuming that skin swelling responses of adults and yearlings involved different components of the immune system, the density-dependent patterns observed in adults suggests a direct negative effect of density on acquired immunity due to increased levels of intraspecific competition at high densities (e.g., Svensson et al. 2001, Tella et al. 2001, Hawley et al. 2006). The absence of a direct effect of density on local inflammation in yearlings could indicate that the maintenance of innate immunity is possible even when resources are limited, in contrast to acquired cell-mediated immunity (e.g., Hangalapura et al. 2005, Bourgeon et al. 2007). A second hypothesis is that population density had contrasting organizational and activational effects on the immune system of growing individuals (yearlings) and of fully grown individuals (adults), respectively. The density independence observed in yearlings could be due to adaptive plasticity during the development of the immune system (e.g., Birkhead et al. 1999, Tschirren et al. 2009), whereas density-dependent changes in adults might represent constraints on a mature immune system due to decreased energy intakes and increased levels of social stress with crowding (e.g., Houston et al. 2007, Edeline et al. 2010).

In accordance with the second hypothesis, we previously described a similar age-dependent plasticity of local inflammation in which food deprivation had positive long-term effects on local inflammation for up to two years when experienced during the juvenile development, but had negative effects for up to one year when experienced later in life (Mugabo et al. 2010, 2011). These differences between age classes were observed after a primary exposure to PHA and therefore could not be attributed to an activation of different components of the immune system between age classes (Mugabo et al. 2010, 2011). In addition, the nonsignificant density dependence of local inflammation in yearlings contrasted with the strong negative density feedbacks on growth and reproduction, but constant survival (described in Mugabo et al. 2013). This could suggest that, in growing lizards, survival is traded against structural growth and reproduction through investment in immune defenses when resources are limited (e.g., Tschirren and Richner 2006, Kopp and Medzhitov 2009).

Furthermore, contrary to our expectation of a greater sensitivity to density in females than in males, local inflammation was similarly affected by density in both sexes. Initially, our prediction was based on our findings that compensatory density dependence of body growth and reproduction was stronger in females than in males (Mugabo et al. 2013). In general, a higher susceptibility of body growth and/or reproduction to adverse conditions in one sex is associated with a higher susceptibility of immune defenses (e.g., Fargallo et al. 2002, Zuk et al. 2004, McKean and Nunney 2005). The absence of sex-specific immune responses instead revealed discrepancies between the plasticity of immunity and other life history traits in common lizards. Such a discrepancy has already been described in this species (Mugabo et al. 2010, 2011). This decoupling could arise from differences in allocation strategies of limited resources to immunity and life history traits, or from differences in their sensitivity to energy shortage and social stress (Tschirren et al. 2003, Mugabo et al. 2010, 2011). 


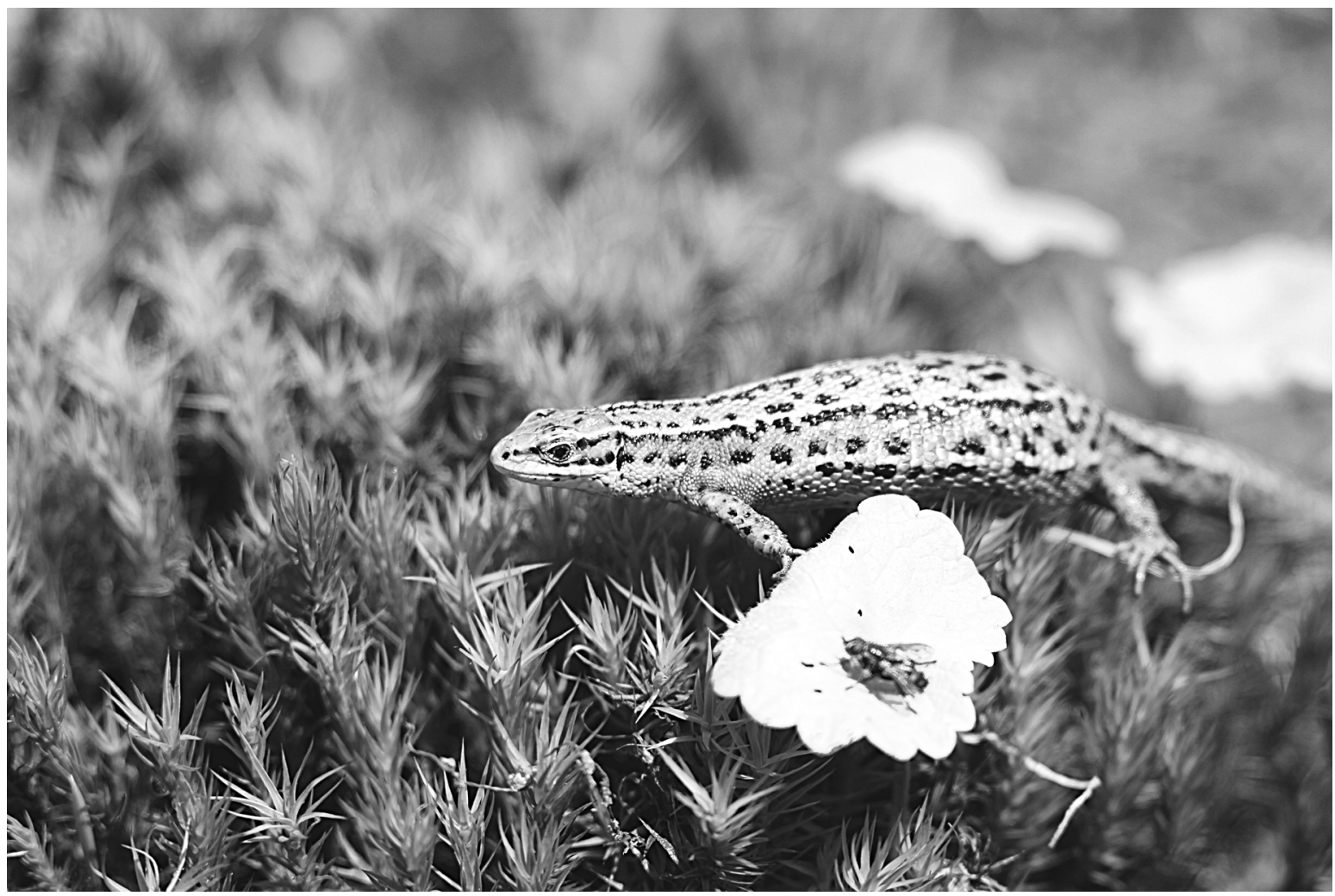

Plate 1. The common lizard (Zootoca vivipara) lives in humid bogs and meadows in cold habitats across Eurasia. As shown in this study, females such as the one photographed here are less infected by ectoparasite ticks especially when they are introduced in high density populations. Photo credit: S. Perret.

\section{Encounter-dilution effect of the risk of infection}

Tick abundance and prevalence were negatively correlated with lizard density. These results demonstrate an encounter-dilution effect of the risk of parasitism by the mobile ectoparasite Ixodes ricinus with lizard density, as defined by Mooring and Hart (1992) at the host intraspecific level. Ixodid ticks are host generalists and are vectors to many pathogens responsible for zoonoses (Wikel 2013). In multiple host-parasite systems, lizards can play an important role in the risk of transmission of tick-borne pathogens by decreasing (e.g., Matuschka et al. 1992, Richter and Matuschka 2006) or increasing (e.g., Richter and Matuschka 2006, Swei et al. 2011) the prevalence or abundance of infected ticks, depending on their reservoir competence and on the proportion of the population of ticks hosted by lizards (Swei et al. 2011). For instance, in the United States, the prevalence of I. pacificus infected by the Lyme disease agent Borrelia burgdorferi decreases with lizard abundance (incompetent hosts of B. burgdorferi), but lizards also support a large proportion of immature ticks. As a result, the abundance of infected nymphal $I$. pacificus increases with lizard abundance, along with the risk of Lyme disease in the host community (Salkeld and Lane 2010, Swei et al. 2011). In Europe, lizard species are important hosts of immature I. ricinus and are incompetent reservoirs for most tick-borne pathogens (Richter and Matuschka 2006, Tijsse-Klasen et al. 2010, Wodecka et al. 2014). As a dominant and abundant species of bogs and heather lands, Z. vivipara could therefore host a large proportion of larval and nymphal I. ricinus and encounter-dilution effects in $Z$. vivipara could affect the risk of transmission of tick-borne pathogens in its natural habitats.

Tick prevalence strongly decreased with lizard density in females, but not in males. Tick abundance was similarly affected by density in both sexes, but showed a weaker pattern of density dependence than prevalence in females (post hoc tests of the effect of density: for prevalence, $\mathrm{LRT}=13.78, P=0.0002$; for abundance, LRT $=4.41, P=0.04$ ). If the encounter-dilution effect were stronger in females than in males, as suggested by prevalence analyses, then a similar sex-specific pattern of density dependence should have been detected in abundance. In addition, if the reduction of parasite exposure with lizard density had a stronger effect at the lower end than at the top end of the range of tick infestation, i.e., at low rather than at high levels of infestation, then a stronger response to density should have been observed in prevalence than in abundance, due to parasite aggregation among hosts (Krasnov et al. 2007). In accordance with this hypothesis, we found that 
abundance decreased with density in weakly infested (abundance below the average of four ticks per host) but not in highly infested (with four or more ticks per host) lizards (Appendix D). This result suggests that the reduction in the risk of parasitism with lizard density influenced low levels of infestation more than high levels of infestation. Differences in the density dependence of resistance and/or exposure to parasites could have contributed to a stronger encounter-dilution effect at low than at high levels of infestation. In females, this could explain the stronger pattern of density dependence observed in prevalence than in abundance data. Conversely, in males, high levels of tick infestation most likely prevented us from detecting the occurrence of an encounter-dilution effect with prevalence data, despite the decrease of the number of ticks per individual with lizard density.

\section{Pathways between host density, host immunity, and host-parasite interactions}

Our path analyses of three alternative scenarios revealed a different relationship in yearlings than in adults. In adults, path analyses suggested that lizard density negatively affected local inflammation and tick abundance (encounter-dilution effect), directly and independently. In yearlings, path analyses instead suggested a direct negative effect of lizard density on tick abundance and a direct negative effect of tick abundance on local inflammation, whereas lizard density had no direct effect on local inflammation. A positive direct effect of tick infestation on local inflammation was expected if yearlings adjusted their investment in immunity to exposure to parasites (e.g., Møller et al. 2001, Tschirren and Richner 2006). Conversely, a negative direct effect of tick infestation on local inflammation would suggest that ticks could modulate immune resistance against blood-feeding parasites in yearlings and/or that costs of parasitism might diminish immune defenses for yearlings (Wikel 2013). For instance, molecules produced by the salivary glands of $I$. ricinus inhibit several components of host innate and adaptive immunity and enhance the transmission and establishment of tick-borne pathogens such as B. burgdorferi (reviewed in Wikel 2013). Path analyses, however, suggested that the resulting indirect positive effect of lizard density on local inflammation in yearlings through the dilution of tick exposure was negligible. In adults, the absence of a significant effect of tick infestation on local inflammation could be due to a resistance to tick saliva immunomodulatory components (Wikel 2013) or to an immunological tolerance to infection acquired through repeated infestations (e.g., Duerr et al. 2004, Schneider and Ayres 2008).

To conclude, lizards do not seem to adjust their investment in local inflammation to the risk of being infected by a specific blood-feeding ectoparasite. This could be due to the generalist role played by inflammatory responses in host defenses against several patho- gens, from ectoparasites to microorganisms, and in wound healing (Demas et al. 2011). Instead, lizards may acquire resistance and/or tolerance to tick downregulation of immune defenses during the ontogeny of the immune system (Duerr et al. 2004, Wikel 2013). Thus, the complex pathways between host density, host immune defenses, and levels of parasite infestation were influenced by age class, which highlights the need to account for relevant individual traits in order to unravel the mechanisms generating variation in host immunity and host-parasite interactions.

\section{ACKNOWLEDGMENTS}

We are thankful to M. Paquet and field assistants for their support. We also thank T. J. M. Van Dooren, who helped improve a previous version of the manuscript. This study was funded by the Centre National de la Recherche Scientifique (CNRS), the Agence Nationale de la Recherche (ANR, grant 07-JCJC-0120 to J.-F. Le Galliard) and the Région Île-deFrance R2DS program (grant 2007-06 to J.-F. Le Galliard and M. Mugabo). Protocols were done under the agreement with the Regional ethics committee in animal experiment $\mathrm{N}^{\circ} 3$ of the Région Île-de-France (file p3/2008/008).

\section{Literature Cited}

Altizer, S., et al. 2003. Social organization and parasite risk in mammals: integrating theory and empirical studies. Annual Review of Ecology, Evolution, and Systematics 34:517-547.

Amundsen, P. A., R. Knudsen, and A. Klemetsen. 2007. Intraspecific competition and density dependence of food consumption and growth in Arctic charr. Journal of Animal Ecology 76:149-158.

Bates, D. M. 2010. lme4: mixed-effects modeling with R. http:// lme4.r-forge.r-project.org/lMMwR/lrgprt.pdf

Bates, D. M., M. Maechler, and B. M. Bolker. 2013. lme4: Linear mixed-effects models using Eigen and S4. Version 1.05. http://lme4.r-forge.r-project.org/

Birkhead, T. R., F. Fletcher, and E. J. Pellatt. 1999. Nestling diet, secondary sexual traits and fitness in the zebra finch. Proceedings of the Royal Society B 266:385-390.

Bolker, B. M., M. E. Brooks, C. J. Clark, S. W. Geange, J. R. Poulsen, M. H. H. Stevens, and J. S. S. White. 2009. Generalized linear mixed models: a practical guide for ecology and evolution. Trends in Ecology and Evolution 24:127-135.

Bourgeon, S., T. Raclot, Y. Le Maho, D. Ricquier, and F. Criscuolo. 2007. Innate immunity, assessed by plasma NO measurements, is not suppressed during the incubation fast in eiders. Developmental and Comparative Immunology 31: $720-728$.

Brown, G. P., C. M. Shilton, and R. Shine. 2011. Measuring amphibian immunocompetence: validation of the phytohemagglutinin skin-swelling assay in the cane toad, Rhinella marina. Methods in Ecology and Evolution 2:341-348.

Burnham, K. P., D. R. Anderson, and K. P. Huyvaert. 2011. AIC model selection and multimodel inference in behavioral ecology: some background, observations, and comparisons. Behavioral Ecology and Sociobiology 65:23-35.

Christe, P., R. Arlettaz, and P. Vogel. 2000. Variation in intensity of a parasitic mite (Spinturnix myoti) in relation to the reproductive cycle and immunocompetence of its bat host (Myotis myotis). Ecology Letters 3:207-212.

Christe, P., O. Glaizot, G. Evanno, N. Bruyndonckx, G. Devevey, G. Yannic, P. Patthey, A. Maeder, P. Vogel, and R. Arlettaz. 2007. Host sex and ectoparasites choice: preference 
for, and higher survival on female hosts. Journal of Animal Ecology 76:703-710.

Coté, I. M., and R. Poulin. 1995. Parasitism and group size in social animals: a meta-analysis. Behavioral Ecology 6:159165.

Demas, G. E., D. A. Zysling, B. R. Beechler, M. P. Muehlenbein, and S. S. French. 2011. Beyond phytohaemagglutinin: assessing vertebrate immune function across ecological contexts. Journal of Animal Ecology 80:710-730.

Duerr, H. P., K. Dietz, H. Schulz-Key, D. W. Buttner, and M. Eichner. 2004. The relationships between the burden of adult parasites, host age and the microfilarial density in human onchocerciasis. International Journal for Parasitology 34: 463-473.

Edeline, E., T. O. Haugen, F. A. Weltzien, D. Claessen, I. J. Winfield, N. C. Stenseth, and L. A. Vollestad. 2010. Body downsizing caused by non-consumptive social stress severely depresses population growth rate. Proceedings of the Royal Society B 277:843-851.

Elston, D. A., R. Moss, T. Boulinier, C. Arrowsmith, and X. Lambin. 2001. Analysis of aggregation, a worked example: numbers of ticks on red grouse chicks. Parasitology 122:563569.

Faraway, J. 2006. Extending the linear model with R: generalized linear, mixed effects and nonparametric regression models. Chapman and Hall/CRC, Boca Raton, Florida, USA.

Faraway, J. 2013. Changes to the Mixed Effects Models chapters in ELM (Extending the linear model with R). https://www.researchgate.net/publication/253376323 Changes_to the_Mixed_Effects_Models_chapters_in_ELM

Fargallo, J. A., T. Laaksonen, V. Poyri, and E. Korpimaki. 2002. Inter-sexual differences in the immune response of Eurasian kestrel nestlings under food shortage. Ecology Letters 5:95-101.

Fauchald, P., R. Rodven, B. J. Bardsen, K. Langeland, T. Tveraa, N. G. Yoccoz, and R. A. Ims. 2007. Escaping parasitism in the selfish herd: age, size and density-dependent warble fly infestation in reindeer. Oikos 116:491-499.

González-Suárez, M., M. Mugabo, B. Decencière, S. Perret, D. Claessen, and J.-F. Le Galliard. 2011. Disentangling the effects of predator body size and prey density on prey consumption in a lizard. Functional Ecology 25:158-165.

Gray, J. S. 1991. The development and seasonal activity of the tick Ixodes ricinus: a vector of Lyme borreliosis. Review of Medical and Veterinary Entomology 79:323-333.

Grear, D. A., S. E. Perkins, and P. J. Hudson. 2009. Does elevated testosterone result in increased exposure and transmission of parasites? Ecology Letters 12:528-537.

Hangalapura, B. N., M. G. B. Nieuwland, G. D. Reilingh, J. Buyse, H. Van Den Brand, B. Kemp, and H. K. Parmentier. 2005. Severe feed restriction enhances innate immunity but suppresses cellular immunity in chicken lines divergently selected for antibody responses. Poultry Science 84:15201529 .

Hawley, D. M., K. Lindstrom, and M. Wikelski. 2006. Experimentally increased social competition compromises humoral immune responses in house finches. Hormones and Behavior 49:417-424.

Heylen, D. J. A., and E. Matthysen. 2008. Effect of tick parasitism on the health status of a passerine bird. Functional Ecology 22:1099-1107.

Houston, A. I., J. M. McNamara, Z. Barta, and K. C. Klasing. 2007. The effect of energy reserves and food availability on optimal immune defence. Proceedings of the Royal Society B $274: 2835-2842$
Keesing, F., R. D. Holt, and R. S. Ostfeld. 2006. Effects of species diversity on disease risk. Ecology Letters 9:485-498.

Kopp, E. B., and R. Medzhitov. 2009. Infection and inflammation in somatic maintenance, growth and longevity. Evolutionary Applications 2:132-141.

Krasnov, B. R., M. Stanko, and S. Morand. 2007. Host community structure and infestation by ixodid ticks: repeatability, dilution effect and ecological specialization. Oecologia 154:185-194.

Lecomte, J., K. Boudjemadi, F. Sarrazin, K. Cally, and J. Clobert. 2004. Connectivity and homogenisation of population sizes: an experimental approach in Lacerta vivipara. Journal of Animal Ecology 73:179-189.

Lochmiller, R. L., and C. Deerenberg. 2000. Trade-offs in evolutionary immunology: Just what is the cost of immunity? Oikos 88:87-98.

Martin, L. B., P. Han, J. Lewittes, J. R. Kuhlman, K. C. Klasing, and M. Wikelski. 2006. Phytohemagglitinin-induced skin swelling in birds: histological support for a classic immunoecological technique. Functional Ecology 20:290299.

Massot, M., J. Clobert, T. Pilorge, J. Lecomte, and R. Barbault. 1992. Density dependence in the common lizard: demographic consequences of a density manipulation. Ecology 73:1742-1756.

Matuschka, F. R., P. Fischer, M. Heiler, D. Richter, and A. Spielman. 1992. Capacity of European animals as reservoir hosts for the Lyme disease spirochete. Journal of Infectious Diseases 165:479-483.

McKean, K. A., and L. Nunney. 2005. Bateman's principle and immunity: phenotypically plastic reproductive strategies predict changes in immunological sex differences. Evolution 59:1510-1517.

Mejlon, H. 2000. Host-seeking activity of Ixodes ricinus in relation to the epidemiology of Lyme borreliosis in Sweden. Dissertation. University of Uppsala, Uppsala, Sweden.

Meylan, S., M. Richard, S. Bauer, C. Haussy, and D. Miles. 2013. Costs of mounting an immune response during pregnancy in a lizard. Physiological and Biochemical Zoology 86:127-136.

Møller, A. P., S. Merino, C. R. Brown, and R. J. Robertson. 2001. Immune defense and host sociality: a comparative study of swallows and martins. American Naturalist 158: $136-145$

Møller, A. P., and N. Saino. 2004. Immune response and survival. Oikos 104:299-304.

Mooring, M. S., and B. L. Hart. 1992. Animal grouping for protection from parasites: selfish herd and encounter dilution effects. Behaviour 123:173-193.

Mugabo, M., O. Marquis, S. Perret, and J.-F. Le Galliard. 2010. Immediate and delayed life history effects caused by food deprivation early in life in a short-lived lizard. Journal of Evolutionary Biology 23:1886-1898.

Mugabo, M., O. Marquis, S. Perret, and J.-F. Le Galliard. 2011. Direct and socially-mediated effects of food availability late in life on life-history variation in a short-lived lizard. Oecologia 166:949-960.

Mugabo, M., S. Perret, S. Legendre, and J.-F. Le Galliard. 2013. Density-dependent life history and the dynamics of small populations. Journal of Animal Ecology 82:12271239.

Owen, J. P., M. E. Delany, C. J. Cardona, A. A. Bickford, and B. A. Mullens. 2009. Host inflammatory response governs fitness in an avian ectoparasite, the northern fowl mite (Ornithonyssus sylviarum). International Journal for Parasitology 39:789-799. 
Pedersen, A. B., and S. A. Babayan. 2011. Wild immunology. Molecular Ecology 20:872-880.

R Development Core Team. 2013. R version 3.0.2. R: A language and environment for statistical computing. $\mathrm{R}$ Foundation for Statistical Computing, Vienna, Austria. http://cran.r-project.org/

Raffel, T. R., J. T. Hoverman, N. T. Halstead, P. J. Michel, and J. R. Rohr. 2010. Parasitism in a community context: traitmediated interactions with competition and predation. Ecology 91:1900-1907.

Richter, D., and F. R. Matuschka. 2006. Perpetuation of the Lyme disease spirochete Borrelia lusitaniae by lizards. Applied and Environmental Microbiology 72:4627-4632.

Rifkin, J. L., C. L. Nunn, and L. Z. Garamszegi. 2012. Do animals living in larger groups experience greater parasitism? A meta-analysis. American Naturalist 180:70-82.

Salkeld, D. J., and R. S. Lane. 2010. Community ecology and disease risk: lizards, squirrels, and the Lyme disease spirochete in California, USA. Ecology 91:293-298.

Schneider, D. S., and J. S. Ayres. 2008. Two ways to survive infection: what resistance and tolerance can teach us about treating infectious diseases. Nature Reviews Immunology 8: 889-895.

Sheldon, B. C., and S. Verhulst. 1996. Ecological immunology: costly parasite defences and trade-offs in evolutionary ecology. Trends in Ecology and Evolution 11:317-321.

Shipley, B. 2000. Cause and correlation in biology: a user's guide to path analysis, structural equations and causal inference. Cambridge University Press, Cambridge, UK.

Shipley, B. 2009. Confirmatory path analysis in a generalized multilevel context. Ecology 90:363-368.

Simkova, A., D. Kadlec, M. Gelnar, and S. Morand. 2002. Abundance-prevalence relationship of gill congeneric ectoparasites: testing the core satellite hypothesis and ecological specialisation. Parasitology Research 88:682-686.

Svensson, E., B. Sinervo, and T. Comendant. 2001. Densitydependent competition and selection on immune function in genetic lizard morphs. Proceedings of the National Academy of Sciences USA 98:12561-12565.

Swei, A., R. S. Ostfeld, R. S. Lane, and C. J. Briggs. 2011. Impact of the experimental removal of lizards on Lyme disease risk. Proceedings of the Royal Society B 278:29702978.

Tella, J. L., M. G. Forero, M. Bertellotti, J. A. Donazar, G. Blanco, and O. Ceballos. 2001. Offspring body condition and immunocompetence are negatively affected by high breeding densities in a colonial seabird: a multiscale approach. Proceedings of the Royal Society B 268:14551461.
Tijsse-Klasen, E., M. Fonville, J. H. J. Reimerink, A. Spitzenvan der Sluijs, and H. Sprong. 2010. Role of sand lizards in the ecology of Lyme and other tick-borne diseases in the Netherlands. Parasites and Vectors 3:42.

Trenzado, C. E., A. E. Morales, and M. de la Higuera. 2006. Physiological effects of crowding in rainbow trout, Oncorhynchus mykiss, selected for low and high stress responsiveness. Aquaculture 258:583-593.

Tschirren, B., L. L. Bischoff, V. Saladin, and H. Richner. 2007. Host condition and host immunity affect parasite fitness in a bird-ectoparasite system. Functional Ecology 21:372-378.

Tschirren, B., P. S. Fitze, and H. Richner. 2003. Sexual dimorphism in susceptibility to parasites and cell-mediated immunity in great tit nestlings. Journal of Animal Ecology 72:839-845.

Tschirren, B., and H. Richner. 2006. Parasites shape the optimal investment in immunity. Proceedings of the Royal Society B 273:1773-1777.

Tschirren, B., A. N. Rutstein, E. Postma, M. Mariette, and S. C. Griffith. 2009. Short- and long-term consequences of early developmental conditions: a case study on wild and domesticated zebra finches. Journal of Evolutionary Biology 22:387-395.

Turmelle, A. S., J. A. Ellison, M. T. Mendonca, and G. F. McCracken. 2010. Histological assessment of cellular immune response to the phytohemagglutinin skin test in Brazilian free-tailed bats (Tadarida brasiliensis). Journal of Comparative Physiology B 180:1155-1164.

Viljoen, H., N. C. Bennett, E. A. Ueckermann, and H. Lutermann. 2011. The role of host traits, season and group size on parasite burdens in a cooperative mammal. PLoS ONE 6(11):e27003.

Vinkler, M., H. Bainova, and T. Albrecht. 2010. Functional analysis of the skin-swelling response to phytohaemagglutinin. Functional Ecology 24:1081-1086.

Wikel, S. 2013. Ticks and tick-borne pathogens at the cutaneous interface: host defenses, tick countermeasures, and a suitable environment for pathogen establishment. Frontiers in Microbiology 4:337.

Wodecka, B., A. Rymaszewska, and B. Skotarczak. 2014. Host and pathogen DNA identification in blood meals of nymphal Ixodes ricinus ticks from forest parks and rural forests of Poland. Experimental and Applied Acarology 62:543-555.

Zuk, M., L. W. Simmons, J. T. Rotenberry, and A. M. Stoehr. 2004. Sex differences in immunity in two species of field crickets. Canadian Journal of Zoology 82:627-634.

\section{Supplemental Material}

\section{Ecological Archives}

Appendices A-D are available online: http://dx.doi.org/10.1890/14-0524.1.sm 